\title{
Producción científica iberoamericana sobre TIC en el contexto educativo
}

\section{Iberoamerican scientific production on ICT in the educational context}

\author{
Ronald M. Hernandez* \\ Universidad Católica Santo Toribio de Mogrovejo, Chiclayo, Perú \\ ORCID: https://orcid.org/0000-0003-1263-2454 \\ Miguel A. Saavedra-López \\ Universidad Continental, Cusco, Perú \\ ORCID: https://orcid.org/0000-0003-4913-933X \\ Emma M. Wong-Fajardo \\ Universidad Católica Santo Toribio de Mogrovejo, Chiclayo, Perú \\ ORCID: https://orcid.org/0000-0002-3775-379X \\ Osmer Campos-Ugaz \\ Universidad Católica Santo Toribio de Mogrovejo, Chiclayo, Perú \\ ORCID: http://orcid.org/0000-0002-3876-6605
}

Xiomara M. Calle-Ramírez

Universidad Nacional de Tumbes, Tumbes, Perú

ORCID: https://orcid.org/0000-0002-7773-1800

Mayra V. García-Pérez

Universidad Continental, Cusco, Perú

ORCID: https://orcid.org/0000-0003-1996-8495

*Correspondencia:

Email: ronald.hernandez@outlook.com.pe

\section{Citar como:}

Hernandez, R., Saavedra-López, M., Wong-Fajardo, E., Campos-Ugaz, O., Calle-Ramírez, X., \& García-Pérez, M. (2021). Producción científica iberoamericana sobre TIC en el contexto educativo. Propósitos y Representaciones, 9(3), e1443. http://dx.doi.org/10.20511/pyr2021.v9n3.1443 


\section{Resumen}

Las TIC en el contexto educativo pueden verse conocidos como recursos transmisores de información que vincula al docente y al estudiante en la mejora de la enseñanza - aprendizaje adaptado a las necesidades y características de cada sujeto. Desde esta perspectiva se busca describir el estado de la producción científica Iberoamericana sobre la inclusión de las TIC en la disciplina educativa en el periodo 2010 al 2020. El estudio se basa en el análisis descriptivo retrospectivo de publicaciones recopiladas en revistas indizadas en Scopus. Se recolectó un total de $n=1976$ contribuciones. Se encontró que la producción aumentó en un 8,51\%. España es el país Iberoamericano que contribuye con mayor producción científica, los autores con afiliación Iberoamericana suelen publicar a través de Artículos de conferencia, destacando ACM International Conference Proceeding Series. Frente a un análisis de madurez de conocimiento estamos ante un desarrollo de producción científica en proceso de crecimiento.

Palabras clave: Conocimiento; Tecnología de Información y Comunicación; Educación; Iberoamérica; Producción científica; Análisis de contenido.

\section{Summary}

ICT in the educational context can be seen as information transmitting resources that link the teacher and the student in the improvement of teaching-learning adapted to the needs and characteristics of each subject. From this perspective, we seek to describe the state of Iberoamerican scientific production on the inclusion of ICT in the educational discipline in the period 2010 to 2020 , based on a retrospective descriptive analysis of publications collected in journals indexed in Scopus. A total of $n=1976$ contributions were collected. It was found that production increased by $8.51 \%$. Spain is the Iberoamerican country that contributes with the highest scientific production, authors with Iberoamerican affiliation usually publish through conference articles, highlighting ACM International Conference Proceeding Series. In front of an analysis of maturity of knowledge we are facing a development of scientific production in process of growth.

Keywords: Knowledge; Information and Communication Technology; Education; Iberoamerica; Scientific production; Content analysis.

\section{Introducción}

La Sociedad de la información, en la cual vivimos, nos ha facilitado una serie de actividades y soluciones a problemas de distinta índole, haciendo uso del acceso, manejo e intercambio de contenidos virtuales (Alfonso, 2016; González et al., 2018). Los avances en las Tecnologías de la Información y la Comunicación (TIC) han tenido, durante los últimos años, un desarrollo notable en el campo educativo convirtiéndose en uno de los principales desafíos de la sociedad actual (Martín, 2017; Vinueza \& Simbaña, 2017) es así que la incorporación de las TIC ha significado para los espacios educativos una iniciativa para el acceso a la información aprovechando las tecnologías en los procesos formativos de enseñanza y aprendizaje (Islas, 2017).

Las TIC han cambiado las relaciones educativas entre el docente y el estudiante, dado que las diversas escuelas están afrontando la educación de la llamada generación $\mathrm{Z}$ y la incorporación del estudiante a la sociedad digital y es aquí, donde surge la idea de señalar como un nuevo elemento de la formación pedagógica la relación del docente y la tecnología educativa (Gutiérrez \& Torrego, 2018; Popescu et al., 2019).

Investigaciones previas como que los cambios en la formación docente en el uso de TIC revelan un efecto favorable en la enseñanza (Popescu et al., 2019; Zempoalteca et al., 2017). Por 
otro lado, las dimensiones de la competencia digital que se relacionan más con la formación docente coinciden en la resolución de problemas referida expresamente al uso de recursos TIC (Islas, 2017). Lipsman (2016), por su parte, plantea que los propósitos pedagógicos subyacen las propuestas que incorporan TIC en la enseñanza. Ruiz et al. (2018) indican que las TIC en el contexto educativo no garantizan el éxito escolar, sino va acompañado de una metodología adecuada. Otra de las potencialidades de las TIC es destacar la capacidad que tienen para adaptarse a las necesidades del estudiante (Muñoz \& Cubo, 2019).

La revisión de la literatura sobre la inclusión de las TIC en la educación centra su atención en potenciar la formación del docente sobre TIC (Prendes et al., 2010; Hernández \& Torrijos, 2019). Frente a esto, los estudios de producción científica resultan relevantes en el análisis del conocimiento en disciplinas y subdisciplinas (Corchuelo, 2018). El auge de estos estudios también es concomitante con el crecimiento de la producción científica de un país (Maz-Machado et al., 2016). En esta tarea, las instituciones de educación superior son las que encabezan la producción de documentos científicos (Bucheli et al., 2012). El análisis de la producción generada al interior de las instituciones de educación superior y centros de desarrollo de la ciencia representa una tarea necesaria para ponderar la calidad del trabajo científico, construir redes nacionales e internacionales y crear colaboración institucional entre autores. A partir de lo mencionado, el presente estudio tiene como objetivo describir el estado de la literatura científica sobre la inclusión de las TIC en el contexto educativo.

\section{Método}

Estudio de tipo descriptivo retrospectivo, consideró como unidad de análisis las publicaciones sobre TIC en el contexto educativo, en revistas indizadas en Scopus, durante el periodo 2010 al 2020 y cuya autoría hace mención a afiliaciones de instituciones Iberoamericanas. Scopus incluye más de 40804 revistas de ciencia, tecnología, ciencias sociales, artes, humanidades y medicina, por ello se decidió emplear esta base de datos debido a la gran cantidad de revistas que incluyen, y a su riguroso proceso de selección de revistas, lo cual permite considerar los estudios más relevantes sobre el tema. Además, se le atribuye a Scopus ventajas como la facilidad de navegación, incluye el 100\% de lo indexado en las bases MEDLINE, EMBASE y COMPEDEX, y otras, facilitando el acceso a documentos citados, estando abierta a Internet, disponibilidad de páginas web y patentes (Burham, 2006). La búsqueda incluyó a todos los artículos publicados e indizados, usando los campos Article Title, Abstracts, Keywords, utilizando en los términos de búsqueda las palabras: "New Technologies", "Information and Communication Technologies", " ICT", "Educational Technology", "Technological Education", "Educational Technology" y "Education". Debido al uso exclusivo de la base de datos Scopus y los distintos estudios abordados durante los últimos años y al gran interés que se tiene para la elaboración de mapas temáticos, se debiera usar descriptores y palabras clave de los trabajos publicados en idioma original de la base de datos que los alberga (Callon et al., 1995).

Con los documentos extraídos se organizó en Microsoft Excel una database que incluyó los siguientes datos: nombre de los autores firmantes, título de la publicación, tipo de publicación, instituciones de filiación de los autores firmantes, revista de publicación y país de edición. Finalmente, con apoyo del software VOSviewer se elaboró una red con los principales ejes temáticos asociados a las palabras claves de las publicaciones.

\section{Resultados}

Se encontró un total de 1976 artículos publicados e indizados en Scopus, con autoría a afiliaciones de instituciones iberoamericanas. Se incluyeron en el análisis 07 tipos de documentos publicables. Con mayor porcentaje, el $66.40 \%$ de documentos son artículos de investigación (Tabla 1). 
Tabla 1.

Tipos de documentos de las publicaciones sobre TIC en el contexto educativo

\begin{tabular}{lll}
\hline Tipo de documento & Documentos & $\%$ \\
\hline Artículo & 1312 & 66.40 \\
Artículo de conferencia & 462 & 23.38 \\
Revisión & 91 & 4.61 \\
Capítulo de libro & 79 & 4.00 \\
Libro & 20 & 1.01 \\
Editorial & 8 & 0.40 \\
Notas & 4 & 0.20 \\
\hline
\end{tabular}

$\%=$ Frecuencia.

Desde el 2010 al 2020 los artículos sobre TIC en educación han ido aumentando notablemente siendo el último año el que contiene la mayor producción científica sobre esta temática, evidenciando que durante el periodo estudiado la producción aumentó en un 8,51\% (Figura 1)

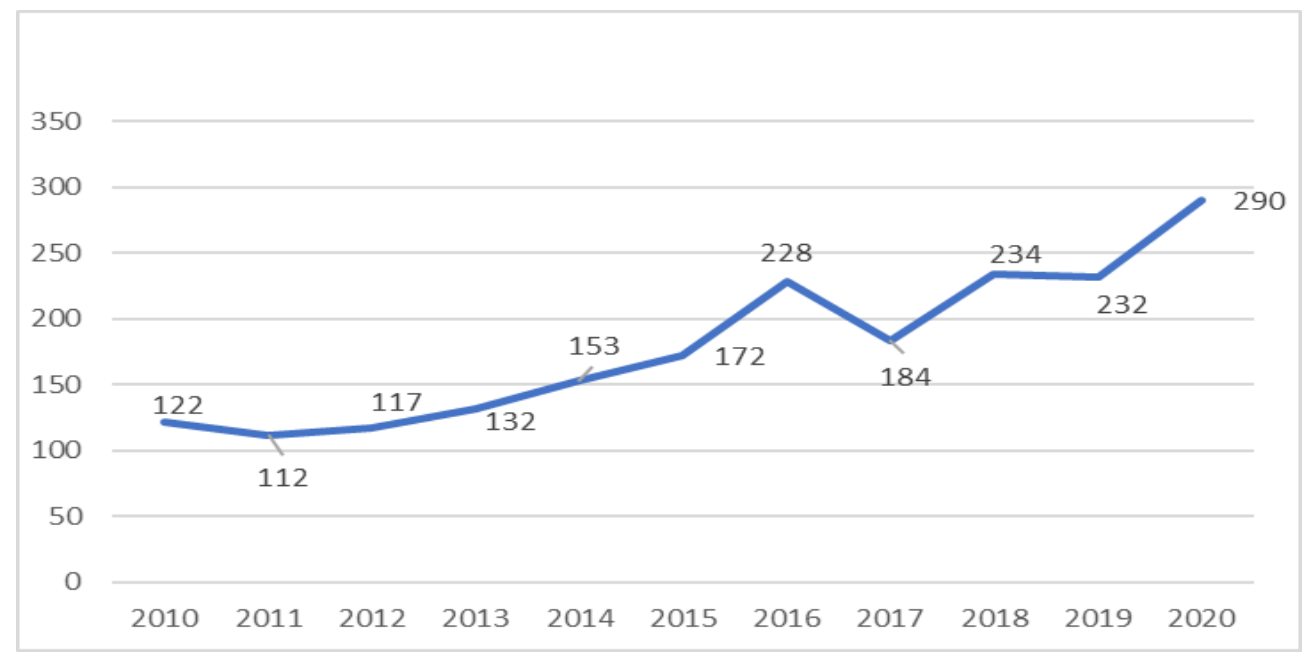

Figura 1. Producción científica sobre TIC en educación distribuida por años

España es el país iberoamericano que contribuye con mayor producción científica sobre TIC y educación, representa el 46,41 \% de la producción iberoamericana, seguido de Brasil, Portugal, Colombia y México, países que sobrepasan el 5,00 \% de la producción. Existen 03 países (República Dominicana, Panamá y Paraguay) que cuentan con al menos 01 publicación durante el periodo estudiado (Tabla 2).

Tabla 2.

Países iberoamericanos con producción científica sobre TIC en el contexto educativo

\begin{tabular}{lll}
\hline País & Documentos & $\%$ \\
\hline España & 917 & 46.41 \\
Brasil & 283 & 14.32 \\
Portugal & 212 & 10.73 \\
Colombia & 135 & 6.83 \\
México & 133 & 6.73 \\
Chile & 94 & 4.76 \\
Ecuador & 76 & 3.85 \\
Argentina & 35 & 1.77 \\
Cuba & 29 & 1.47 \\
Perú & 23 & 1.16
\end{tabular}




$\begin{array}{llr}\text { Venezuela } & 12 & 0.61 \\ \text { Uruguay } & 9 & 0.46 \\ \text { Costa Rica } & 7 & 0.35 \\ \text { Jamaica } & 3 & 0.15 \\ \text { Puerto Rico } & 3 & 0.15 \\ \text { República Dominicana } & 2 & 0.10 \\ \text { Panamá } & 2 & 0.10 \\ \text { Paraguay } & 1 & 0.05\end{array}$

En cuanto a la productividad por institución, 152 instituciones internacionales han participado en la producción iberoamericana sobre TIC en el contexto educativo; se presenta el listado de las 11 primeras, entre los que se destacan las instituciones de España y Brasil, además el $100 \%$ de estas instituciones se ubican dentro de las primeras 100 instituciones del Ranking Iberoamericano de Instituciones de Educación Superior, 2020 (SJR Iber 2020) (Tabla 3).

Tabla 3.

Instituciones iberoamericanas que participan en la investigación sobre TIC y educación

\begin{tabular}{lllc}
\hline Institución & SIR Iber 2020 & País & Documentos \\
\hline Universidad de Salamanca & 46 & España & 106 \\
Universidad de Granada & 14 & España & 72 \\
Universidad de Sevilla & 20 & España & 69 \\
Universitat de València & 12 & España & 61 \\
Universitat Oberta de Catalunya & 18 & España & 55 \\
Universitat de Barcelona & 5 & España & 50 \\
Universidad Nacional de Educación a & 94 & España & 46 \\
Distancia & & & \\
Universidad Complutense de Madrid & 10 & España & 43 \\
Universidad de Murcia & 49 & España & 39 \\
Universidad de Castilla-La Mancha & 50 & España & 39 \\
Universidade de São Paulo & 1 & Brasil & 34 \\
\hline
\end{tabular}

En la Tabla 4 se presenta el listado de las 10 publicaciones más productivas, entre las que se destaca ACM International Conference Proceeding y Digital Education Review (con artículos mayores a 40 documentos). Las áreas temáticas de estas 10 publicaciones son Ciencias de la computación y ciencias sociales. La producción científica está concentrada en revistas de España, lo que demuestra que investigadores de esta región prefieren producir y dar a conocer sus investigaciones en su propio entorno.

Tabla 4.

Publicaciones más productivas sobre TIC en educación

\begin{tabular}{llllc}
\hline Revista & País & $\begin{array}{l}\text { Cuartil } \\
\text { SJR }\end{array}$ & Área temática & Documentos \\
\hline $\begin{array}{l}\text { ACM International } \\
\begin{array}{l}\text { Conference Proceeding } \\
\text { Series }\end{array}\end{array}$ & Estados Unidos & - & $\begin{array}{l}\text { Ciencias de la } \\
\text { computación }\end{array}$ & 46 \\
Digital Education Review & España & Q3 & $\begin{array}{l}\text { Ciencias de la } \\
\text { computación y }\end{array}$ & 43 \\
& & & Ciencias Sociales & \\
Profesorado & España & Q3 & Ciencias sociales & 38 \\
$\begin{array}{l}\text { Comunicar } \\
\text { Revista de Educación }\end{array}$ & España & Q1 & Ciencias sociales & 31 \\
$\begin{array}{l}\text { Risti Revista Iberica de } \\
\text { Sistemas e Tecnologias de }\end{array}$ & Portugal & Q2 & Ciencias sociales & 31 \\
$\begin{array}{l}\text { Informacao } \\
\text { Ceur Workshop Proceedings }\end{array}$ & Estados Unidos & - & Ciencias de la & 31 \\
& & & Ciencias de la & 28
\end{tabular}




\begin{tabular}{|c|c|c|c|c|}
\hline Computers and Education & Reino Unido & Q1 & $\begin{array}{l}\text { Ciencias de la } \\
\text { computación y } \\
\text { Ciencias Sociales }\end{array}$ & 26 \\
\hline Opcion & Venezuela & $\begin{array}{l}\text { Disconti } \\
\text { nuada }\end{array}$ & $\begin{array}{l}\text { Artes y } \\
\text { Humanidades y } \\
\text { Ciencias sociales }\end{array}$ & 24 \\
\hline $\begin{array}{l}\text { Revista Complutense de } \\
\text { Educacion }\end{array}$ & España & Q2 & Ciencias Sociales & 23 \\
\hline
\end{tabular}

En la Tabla 5 se evidencia los autores que han aportado la mayor cantidad de estudios sobre TIC en el contexto educativo. De los 148 autores destacan investigadores como GarcíaPeñalvo, Almerich, Gonzalo y Cabero-Almenara con igual o más de 9 documentos publicados. Dentro de la lista de los 10 principales autores el 50\% está suscrito a una afiliación institucional de España.

Tabla 5.

Autores iberoamericanos con mayor producción de documentos sobre TIC en educación

\begin{tabular}{|c|c|c|c|c|}
\hline Autor & Institución & País & $\mathrm{H}$ index & Documentos \\
\hline $\begin{array}{l}\text { García-Peñalvo, } \\
\text { Francisco José }\end{array}$ & Universidad de Salamanca & España & 38 & 14 \\
\hline Almerich, Gonzalo & Universitat de València, & España & 13 & 9 \\
\hline $\begin{array}{l}\text { Cabero-Almenara, } \\
\text { Julio }\end{array}$ & Universidad de Sevilla, & España & 16 & 9 \\
\hline $\begin{array}{l}\text { Gil, Henrique } \\
\text { Manoel Pires } \\
\text { Teixeira }\end{array}$ & Instituto Politécnico de Castelo & Portugal & 2 & 8 \\
\hline $\begin{array}{l}\text { Rodríguez-Conde, } \\
\text { María José }\end{array}$ & Universidad de Salamanca & España & 10 & 8 \\
\hline Chiappe, Andres & Universidad de La Sabana & Colombia & 6 & 7 \\
\hline Díaz-García, Isabel & Universitat de València & España & 6 & 7 \\
\hline Escudeiro, Paula & $\begin{array}{l}\text { Instituto Superior de Engenharia } \\
\text { do Porto }\end{array}$ & Portugal & 6 & 6 \\
\hline $\begin{array}{l}\text { Guillén-Gámez, } \\
\text { Francisco D. }\end{array}$ & $\begin{array}{l}\text { Universidad Nacional de } \\
\text { Educacion a Distancia }\end{array}$ & España & 7 & 6 \\
\hline Mena, Juanjo & Universidad de Salamanca & España & 11 & 6 \\
\hline
\end{tabular}

En la Figura 2 se observa que el descriptor de mayor frecuencia es Educación con 334 apariciones. En este caso, el número de coocurrencias de dos o más palabras indica el número de publicaciones en el cual los términos aparecen en la lista de las palabras clave de los documentos seleccionados. Los colores indican agrupaciones de palabras clave relativamente relacionadas entre sí según la fortaleza de asociación obtenida por el programa VOSviewer, además de la diferencia visual de las agrupaciones.

Con los 136 descriptores seleccionados de un total de 6595 registrados en los 1976 documentos recuperados se evidencia la agrupación de cinco clústers. El clúster 1 (rojo) incluye la relación de palabras clave entre la innovación educativa a través del uso de las herramientas TIC en el contexto de la educación superior. El clúster 2 (verde) muestra la implicancia de las TIC en la educación y los procesos de enseñanza y las nuevas prácticas docentes. El clúster 3 (azul) analiza las prácticas investigativas sobre la educación tecnológica, el internet y los medios de comunicación social desde el punto de vista de las diferentes disciplinas. El clúster 4 (amarillo) evalúa los distintos componentes de la educación a distancia hacienda uso de herramientas tecnológicas, dispositivos móviles y plataformas virtuales. El clúster 5 (morado) representa la relación del aprendizaje colaborativo y la transmisión de información, la interoperatividad con la web 2.0 


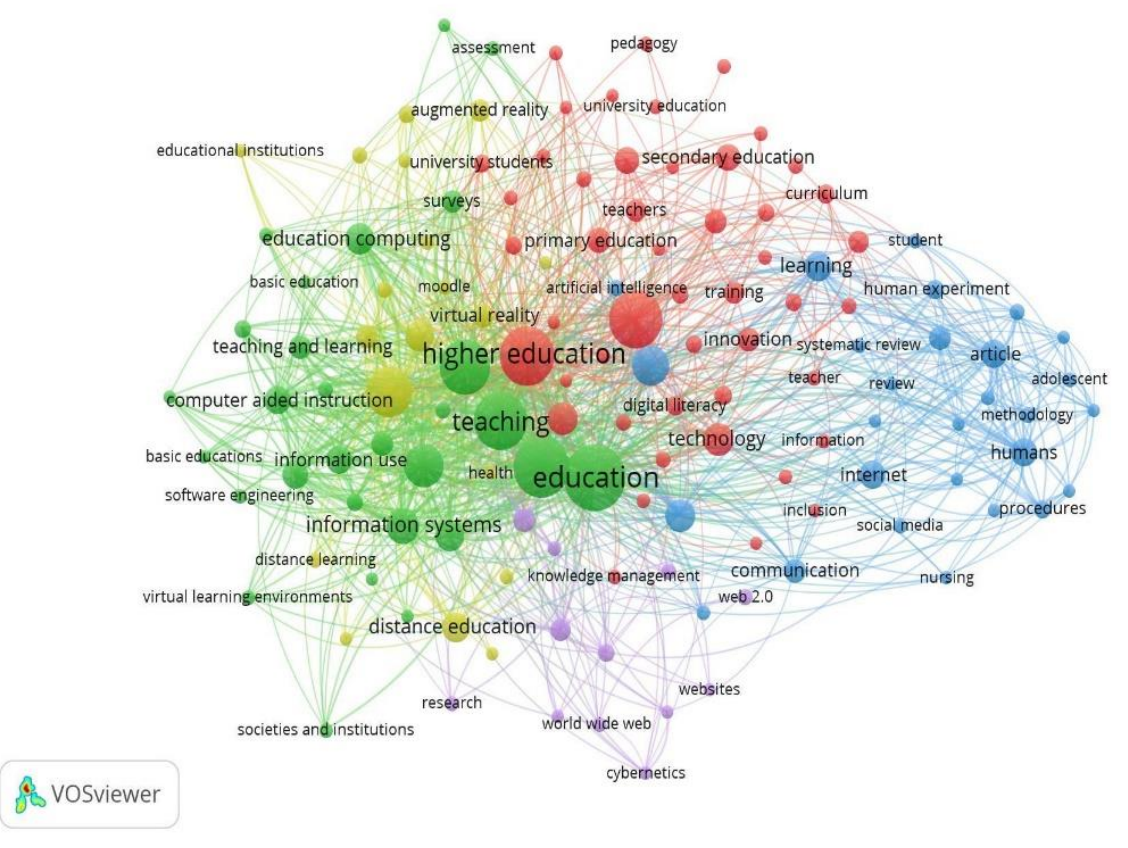

Figura 2. Visualización de una red de aparición de palabras clave

\section{Discusión}

En la actualidad diversos países del mundo han incorporado las tecnologías de la información y comunicación en la educación, incrementando su uso durante la pandemia de Covid-19 por el desarrollo de las clases virtuales. La evolución de las TIC hacia el desarrollo de plataformas de integración ha generado avances en la sociedad actual (Colás \& De Pablos, 2012; PejanovićDjurišić et al., 2017) brindándonos oportunidades educativas, como el desarrollo de clases virtuales y siendo una excelente opción ante la educación tradicional. Por lo mencionado, es importante conocer sobre las investigaciones que se han desarrollado en Iberoamérica respecto a las TIC en el contexto educativo.

De las investigaciones desarrolladas en Iberoamérica, España es el país con mayor número de estudios sobre TIC en el contexto educativo, seguido por Brasil, Portugal, Colombia y México. Resultados similares se encontraron en un estudio realizado en idioma español durante los años 1984 a 2018, donde en las primeras posiciones se encuentran España y México con el $57 \%$ de la producción científica, seguidas por Colombia (Lorenzo-Lledó et al., 2019). Los resultados se relacionan con el Ranking Iberoamericano de Instituciones de Educación Superior 2021, donde los países con mayor producción científica son España, Brasil, Portugal y México (De-Moya-Anegón et al., 2021). Estos resultados también podrían deberse a que España ha realizado un gran esfuerzo por implementar políticas locales y nacionales con la finalidad de integrar las TIC en la educación, generando cambios y transformaciones en el sistema educativo (Area, 2010).

Durante los años 2010 al 2020 se evidencia un crecimiento de la producción científica sobre TIC en educación, especialmente en el último año se incrementó de manera notable la producción científica sobre esta temática. En el estudio producción académica sobre comunicación en España e Hispanoamérica, concluyen que en la actualidad las TIC han ganado mucho protagonismo, en cuanto al desarrollo de investigaciones (Piñeiro \& Morais, 2019). Resultados similares se encontraron en un estudio sobre la producción científica del impacto de TIC sobre las habilidades de escritura y lectura en los años 2010-2020, concluyen que se 
incrementó la producción de investigaciones en los últimos años (Batanero et al., 2021). Asimismo, en un estudio sobre la producción científica de alfabetización digital a nivel universitario publicada en el Scopus, se encontró un incremento en los años 2019 y 2020 (Caldevilla-Dominguez et al., 2021). En la actualidad existe interés de los investigadores respecto a estudiar sobre los conocimientos de los docentes y estudiantes de los diferentes niveles educativos sobre las habilidades que poseen para afrontar los nuevos entornos de aprendizaje vinculados al uso de las TIC, este planteamiento fundamentaría el incremento de las publicaciones en los últimos años (Luna-Romero et al., 2019).

Respecto a la producción científica por instituciones, se encontró que 152 instituciones internacionales han participado en la producción iberoamericana sobre TIC en el contexto educativo; encontrándose que dentro las instituciones que participan más en investigación pertenecen a los países de España y Brasil. En el estudio realizado sobre tendencias de la investigación en educación científica en América Latina, se encontró que los autores brasileños son los que desarrollan más publicaciones (Medina-Jerez, 2018). Los resultados podrían deberse que en Latinoamérica sólo Brasil invierte más del 1\% de su Producto Interno Bruto en actividades relacionadas con la investigación (Banco Mundial, 2017); y el resto de los países invirtió menos del $0,70 \%$ de su producto en I+D, asimismo España invierte el 1,20\% (Red de Indicadores de Ciencia y Tecnología -Iberoamericana e Interamericana, 2019).

La mayoría de las investigaciones se encuentran publicadas en revistas de España, dando a conocer que los investigadores españoles prefieren publicar sus estudios en revistas de su propio país. En un estudio realizado sobre el análisis de la productividad y visibilidad en Scopus de los investigadores españoles en Comunicación, se encontró que la mayoría prefiere publicar en idioma español y en revistas de Abadal, plantea que el 26.32\% de las revistas científicas españolas son de acceso abierto, es decir no solicitan pagos por publicar y las editoriales pertenecen a universidades, sociedades científicas, asociaciones y colegios profesionales (Costa-Sánchez, 2017). Asimismo, España se encuentra en el quinto lugar en el ranking de países con revistas en acceso abierto a nivel mundial (Abadal, 2012).

Asimismo, se halló que el descriptor que se utilizó con mayor frecuencia frente a las TIC fue educación. En un estudio bibliométrico sobre el aula invertida en la educación superior, encontraron que el análisis de palabras clave muestra que el aula invertida en la educación superior presenta relación con descriptores como aprendizaje activo, el aprendizaje mixto y TIC (Limaymanta et al., 2021). De igual forma, Cabrera-Ramos (2020) encontró que los principales temas investigados en los últimos años han estado relacionados a la pedagogía, la tecnología, la sociología, la tecnología, la comunicación y la gestión de la integración de las TIC. Asmat Vega, et al. (2019) encontraron que el $26 \%$ de artículos correspondió a variables relacionadas a la "enseñanza virtual" seguido de educación médica y salud pública con un $21 \%$ respectivamente.

A través del análisis de la producción de artículos de investigación realizados, se identificó que existe un mayor nivel de interés en ciertas disciplinas, evidenciándose que existe un interés creciente por temas educativos y su relación con las TIC, de esta manera se abren nuevas líneas de investigación que puedan dar beneficios prácticos a la virtualidad enfocados quizás en un contexto de pandemia o dando ruptura a las brechas de conexión.

\section{Conclusiones}

Los resultados encontrados dan a conocer la importancia de desarrollar estudios bibliométricos, donde se puede conocer sobre los tipos de estudios realizados, la producción por año, investigadores, instituciones participantes y los descriptores más utilizados sobre la producción científica en Iberoamérica sobre las TIC en el contexto educativo. 
En la actualidad existen varias bases de datos donde se pueden publicar estudios relacionados al tema y por ende la investigación podría ser mucho mayor a lo encontrado en el estudio, sin embargo se consideró Scopus porque se encuentran revistas que consideran procesos rigurosos en la selección de artículos, considerándose valioso el estudio ya que se pudo conocer los estudios realizados sobre la variable mencionada, encontrándose 1976 artículos publicados sobre la producción científica iberoamericana sobre TIC en el contexto educativo, siendo la mayoría artículos originales, se encontró un crecimiento de la publicaciones en los últimos años, la mayoría de instituciones y revistas donde se publicaron pertenecen a España, los países que más publicaron fueron España, Brasil y Portugal, estos resultados guardan relación con el Ranking Iberoamericano de Instituciones de Educación Superior 2021, considerándose a España, Brasil, Portugal y México como los países con mayor capacidad de producción científica de Iberoamérica.

Existen otros indicadores de producción científica que no han sido tenidos en cuenta en el presente estudio y que podrían abordarse en futuras, como la profesión de los autores, el índice de impacto de sus trabajos y la financiación de los estudios. Asimismo, otra limitación de este estudio se refiere al hecho de que, solo se consideró la base de datos Scopus, se sugiere para próximos estudios incluir otras bases de datos como Web of Science o SciELO. No obstante, y pese a las limitaciones, este estudio ofrece un panorama actual sobre la investigación de las TIC en el quehacer educativo.

\section{Referencias}

Alfonso, I. (2016). La sociedad de la Información, Sociedad del Conocimiento y sociedad del Aprendizaje. Referentes en torno a su formación. Bibliotecas. Anales de investigación, 12(2), 236-243. http://revistas.bnjm.cu/index.php/BAI/article/view/179

Abadal, E. (2012). Challenges for open access journals: quantity, quality and economic sustainability. Hipertext.net, 10. http://www.upf.edu/hipertextnet/numero-10/retosrevistas-en-acceso-abierto.html

Asmat, N., Borja Villanueva, C., Bernuy Torres, L., Lizarzaburu Aguinaga, D. \& Morillo Flores, J. (2019). Estudio bibliométrico de la producción científica sobre TIC en Perú (20102017). Propósitos y Representaciones, 7(2), 196-202. https://dx.doi.org/10.20511/pyr2019.v7n2.269

Area, M. (2010). El proceso de integración y uso pedagógico de las TIC en los centros educativos. Un estudio de casos. Revista de Educación, 352, 77-98. http://www.revistaeducacion.educacion.es/re352/re352_04.pdf

Batanero, J., Rueda, M., Cerero, J. \& Gravan, P. (2021) Impact of ICT on writing and reading skills: a systematic review (2010-2020). Texto Livre, 14(2), e34055. https://doi.org/10.35699/1983-3652.2021.34055

Bucheli V., Díaz A., Calderón J., Lemoine P., Valdivia J., Villaveces J. \& Zarama R. (2012). Growth of scientific production in Colombian universities: an intellectual capital-based approach. Scientometrics, 91(2), 369-382. https://doi.org/10.1007/s11192-012-0627-7

Burham, J. F. (2006). Scopus database. A review. Biomedical Digital Libraries, 3, 1. https://doi.org/10.1186/1742- 5581-3-1

Callon, M., Courtial, J. \& Penan, H. (1995). Cienciometría. Ediciones TREA

Cabrera-Ramos, J. F. (2020). Producción científica sobre integración de TIC a la Educación Física: estudio bibliométrico en el periodo 1995-2017. Retos: nuevas tendencias en educación física, deporte y recreación, (37), 748-754. https://doi.org/10.47197/retos.v37i37.67348

Caldevilla-Domínguez, D., Martínez-Sala, A. M. \& Barrientos-Báez, A. (2021). Tourism and ICT. Bibliometric Study on Digital Literacy in Higher Education. Education Sciences, 11(4), 172. https://doi.org/10.3390/educsci11040172 
Colás, P. \& De Pablos, J. (2012). Aplicaciones de las tecnologías de la información y la comunicación en la investigación cualitativa. Revista española de pedagogía, 70(251), 77-92. https://revistadepedagogia.org/wp-content/uploads/2012/02/251-05.pdf

Corchuelo C. (2018). Visibilidad científica y académica en la web 2.0 Análisis de grupos de investigación de la Universidad de La Sabana. Información, Cultura y Sociedad, 38, 7788. https://doi.org/10.34096/ics.i38.3724

Costa-Sánchez, C. (2017). Análisis de la productividad y visibilidad en Scopus de los investigadores españoles en Comunicación. Observatorio, 11(3), 1-16.

De-Moya-Anegón, F., Herrán-Páez, E., Bustos-González, A., Corera-Álvarez, E., TibanáHerrera, G. \& Rivadeneyra, F. (2021). Ranking iberoamericano de instituciones de educación superior 2021 (SIR Iber). Ediciones Profesionales de la Información. https://doi.org/10.3145/sir-iber-2021

González, C., Nieto, Y., Montenegro, C. \& López, J. (2018). Sociedad de la Tecnología la información y el conocimiento: tecnologías en la formación de los ingenieros. Revista Ibérica de Sistemas e Tecnologías de Informaçao, 15(1), 304-317.

Gutiérrez, A. \& Torrego, A. (2018). Educación mediática y su didáctica. Una propuesta para la formación del profesorado en TIC y medios. Revista Interuniversitaria de formación del profesorado, 32(1), 15-27.

Hernández, J.P. \& Torrijos, P. (2019). Percepción del profesorado universitario sobre la integración de las Tecnologías de la Información y la Comunicación (TIC) en las modalidades docentes. Influencia del género y la edad. EDMETIC, 8(1), 128-146. https://doi.org/10.21071/edmetic.v8i1.10537

Islas, C. (2017). La implicación de las TIC en la educación: Alcances, Limitaciones y Prospectiva. RIDE. Revista Iberoamericana para la Investigación y el Desarrollo Educativo, 8(15), 861-876. https://doi.org/10.23913/ride.v8i15.324

Limaymanta, C., Apaza-Tapia, L., Vidal, E. \& Gregorio-Chaviano, O. (2021). Flipped Classroom in Higher Education: A Bibliometric Analysis and Proposal of a Framework for it Implementation. International Journal of Emerging Technologies In Learning, 16(09), 133-149. https://doi.org/10.3991/ijet.v16i09.21267

Lipsman, M. (2016). La innovación con tecnologías en las propuestas de enseñanza de grado. En M. Insaurralde (comp.). La enseñanza en la educación superior. Investigaciones, experiencias y desafíos, 139-156.

Lorenzo-Lledó, G., Lorenzo-Lledó, A. \& Lledó, A. (2019). Las TIC en el contexto educativo a través de la producción científica en español. Revista General de Información y Documentación, 29(2), 287-307. https://doi.org/10.5209/rgid.66969

Luna-Romero, A., Vega, F. \& Carvajal, H. (2019). Formación docente en el uso de las TIC. Revista Universidad, Ciencia y Tecnología, 2(1), 46-52. https://uctunexpo.autanabooks.com/index.php/uct/article/view/66

Martín, M. (2017). Aportaciones pedagógicas de las TIC a los estilos de aprendizaje. Tendencias pedagógicas, (30), 91-104. https://doi.org/10.15366/tp2017.30.005

Maz-Machado, A., Jiménez-Fanjul, N. N. \& Villarraga-Rico, E. (2016). La producción científica colombiana en SciELO: un análisis bibliométrico. Revista Interamericana de bibliotecología, 39(2), 111-119. https://doi.org/10.17533/udea.rib.v39n2a03

Medina-Jerez, W. (2018). Science Education Research Trends in Latin America. International Journal of Science and Mathematics Education, 16, 465-485. https://doi.org/10.1007/s10763-016-9785-z

Muñoz, E., \& Cubo, S. (2019). Competencia digital, formación y actitud del profesorado de educación especial hacia las tecnologías de la información y la comunicación (TIC). Revista de Currículum y Formación del profesorado, 23(1), 210-241. https://doi.org/10.30827/profesorado.v23i1.9151

Pejanović-Djurišić, M., Gavrilovska, L. \& Fratu, O. (2017). Special Issue: ICT Trends for Future Smart World. Wireless Personal Communications, 92(1), 1-3. https://doi.org/10.1007/s11277-016-3886-4 
Piñeiro-Naval, V. \& Morais, R. (2019). Study of the academic production on communication in Spain and Latin America. Media Education Research Journal, 61(27), 108-117. https://www.revistacomunicar.com/html/61/en/61-2019-10.html

Popescu, D., Mariana, D. \& Cotet, B.G. (2019). Preparando a los estudiantes para la Generación Z: consideraciones sobre el currículo de impresión 3D. Propósitos y representaciones, 7(2), 240-268. http://dx.doi.org/10.20511/pyr2019.v7n2.280

Prendes, M., Castañeda, L. \& Gutiérrez, I. (2010). Competences for the use of ICT in future teachers. Comunicar, 35(18), 175-182. https://doi.org/10.3916/C35-2010-03-11

Red de Indicadores de Ciencia y Tecnología -Iberoamericana e Interamericana (2019). El estado de la ciencia: principales indicadores de Ciencia y Tecnología Iberoamericanos / Interamericanos. Editorial Altuna Impresores S.R.L.

Ruiz, A. P., Martínez, A. C. \& Perales, R. G. (2018). Integración de TIC de la Web 2.0 en el campus virtual universitario de la UCLM. (Estudio inter-sujetos). REXE-Revista de Estudios $y$ Experiencias en Educación,2(2), 103-113. http://dx.doi.org/10.21703/rexe.Especial3_20181031139

Vinueza, S. \& Simbaña, V. (2017). Impacto de las TIC en la Educación Superior en el Ecuador. Revista Publicando, 4(11-1), 355-368. http://dx.doi.org/10.47230/unesumciencias.v5.n1.2021.238

Zempoalteca Durán, B., Barragán López, J. F., González Martínez, J. \& Guzmán Flores, T. (2017). Formación en TIC y competencia digital en la docencia en instituciones públicas de educación superior. Apertura (Guadalajara, Jal.), 9(1), 80-96. https://doi.org/10.32870/ap.v9n1.922 